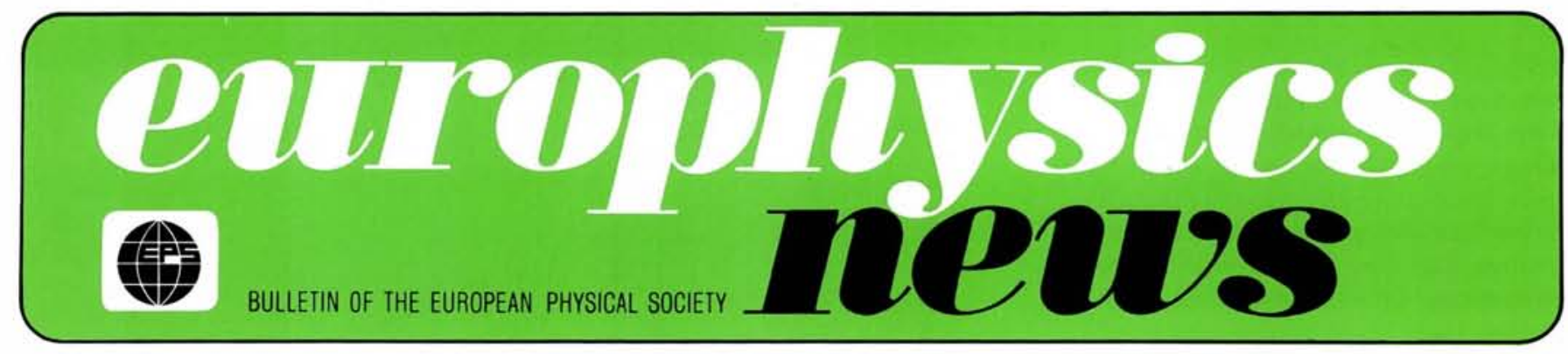

\section{Notice to Members}

As required by the statutes of EPS, members are informed lat least three months in advance) of the intention to ask Council, at its meeting at the end of March 1988 in Dresden, to approve a rise in the unit fee from the current level of Sw.Fr. 11. - to Sw.Fr. 12.-. This would take effect from 1 January 1989 and would mean that independent Individual Ordinary Members would pay an annual membership fee of Sw.Fr. 144. - while IOMs who are members of an EPS national society would pay Sw.Fr. 48. - The national society fees would rise in the same proportion.

The system for changing the unit fee is deliberately cumbersome to encourage stability and the last rise became effective from 1 January 1986. In effect, compensation for three years inflation is proposed at a rate of less than 3\% per year. All costs to the Society have not gone up equally and we have been making hard currency savings through the operations of the Budapest Supplementary Secretariat. Nevertheless we have seen a comfortable operating margin dwindle to a deficit and the Society needs to counteract the trend before it becomes too big.

In the current year the deficit at first sight appears alarming - about Sw.Fr. 80000. - but a significant part of this arises in connexion with extra staff charges necessitated by the unexpectedly high response of authors to the launching of Europhysics Letters. This is not a continuing charge on the Society so the situation can be contained if the rise in the unit fee is agreed.

\section{IOM Delegates}

With this issue of Europhysics News is the membership renewal notice. Please check that it has not been left in the despatch envelope and then deal with it straight away rather than put it to one side where it might get forgotten. The fewer the reminders that have to be sent, the lower the administrative charges on the Society.

No invitation to propose the names of new IOM delegates to Council is included because no vacancies fall due next year. In part, this arises from the change in the standard length of term of office (from three to four years), but more importantly, two serving delegates have been elected to the Executive Committee and so their term is automatically extended.

\title{
Reactivity Coefficients in Nuclear Reactors
}

\author{
M. Hyland, Risley
}

(United Kingdom Atomic Energy Authority)

In the months following the accident at the Chernobyl Nuclear Power Station it became apparent that one of the contributary causes was the 'positive void coefficient of reactivity'. Reactivity coefficients provide a measure of the way in which the neutron multiplication, or reactivity, of a reactor core changes as a function of other reactor variables, such as temperature and pressure, and hence indicate the reactor's inherent stability.

The source of the energy produced in all present day nuclear reactors is the fissioning or splitting up of certain massive nuclei. When such a nucleus is hit by a stray neutron it can absorb it momentarily to form a compound nucleus which then splits up or fissions into two parts, releasing in the process two or three neutrons and a large amount of energy. A nuclear fission reaction typically releases about $200 \mathrm{MeV}$ of energy, associated with a net loss of mass relative to the initial excited compound nucleus. This energy appears mostly in the form of the kinetic energy of the two fission fragments. A typical fission reaction takes the form:

$\mathrm{n}+{ }^{235} \mathrm{U} \rightarrow{ }^{236} \mathrm{U}$

$$
\begin{aligned}
\rightarrow & { }^{141} \mathrm{Ba}+{ }^{92} \mathrm{Kr}+3 \mathrm{n}+ \\
& \text { beta particles }+ \text { neutrinos } \\
& + \text { gamma rays. }
\end{aligned}
$$

Whilst this is only one of many possible ${ }^{235} \mathrm{U}$ fission reactions, each leading to a different pair of fission products, all fission reactions lead to the production of energy and two or three fission neutrons.

It is the production of these neutrons, which in turn can cause further fissions and more neutrons, that leads to the possibility of a self-sustaining or chain reaction, continuously producing large amounts of energy. If, on average, one of the fission neutrons goes on to cause a further fission, the rate of energy production remains constant and the sys- tem is said to be critical. If, however, more than one neutron causes further fissions, the system is said to be supercritical and the rate of energy production increases, while if less than one neutron goes on to cause further fission, the system is said to be sub-critical and the rate of energy production decreases and eventually stops. The state of such systems can be expressed in terms of a multiplication factor, $K$, which is defined as the ratio of the number of neutrons in one generation to the number in the preceeding generation. So

$K<1$ sub-critical power falling $K=1$ critical power constant $K>1$ super-critical power rising The reactivity of a system is defined as:

$$
\rho=(K-1) / K
$$

\section{Contents}

Notice to EPS Members

Reactivity Coefficients in

Nuclear Reactors

Astronomers in Europe, Letter. A \& A Division Replies

Dark Matter in the Universe

Deep Level Point Imperfections in Semiconductors

AMP Divisional Board

Uppsala Conference of

HEPP Division

1987 Nobel Prize Winners

Europhys. News Index for 1987 
and it is thus a measure of the deviation from the condition of criticality $(\rho=0)$.

An important factor in controlling a nuclear reactor is the rate at which the power level changes when the reactivity is non-zero and in particular when it is positive. The time between successive generations of neutrons, that is the average time between the appearance of a fission neutron and the appearance of any further fission neutrons, is typically a millisecond or less. If all neutrons were created at the time of fission, this very short time interval between successive neutron generations would mean that the neutron population and hence the reactor power would change extremely rapidly and would be difficult to control. However, not all neutrons are emitted at the instant of fission. A small fraction - less than $1 \%$ - are emitted by certain fission products which decay by neutron emission rather than by $\beta$ decay. These delayed neutrons appear from a fraction of a second to a few minutes after the fission event and they play a vital role in the control of nuclear reactors. A reactor is designed so that the delayed neutrons are required to keep the chain reaction going and the fact that they do not appear until on average some $10-20$ seconds after fission means that changes in the power level do not occur very rapidly.

If, however, enough reactivity is added, such that the delayed neutrons are no longer necessary to maintain the chain reaction and the prompt neutrons alone can sustain it, then the power level will rise very rapidly and will be extremely difficult to control. This is what happened at Chernobyl.

\section{Controlling the Chain Reaction}

Fission neutrons are emitted with a broad range of energies but, typically, they are in the range of $2-3 \mathrm{MeV}$. In trying to establish the likelihood of one of these 'fast' fission neutrons going on to cause further fission, it is necessary to consider all the possible fates that could befall it. For a uranium fuelled reactor the important events would include:

i) elastic scattering

ii) capture in ${ }^{235} \mathrm{U}$ without fission

iii) capture in ${ }^{235} \mathrm{U}$ with fission

iv) capture in ${ }^{238} \mathrm{U}$ without fission

v) capture in ${ }^{238} \mathrm{U}$ with fission

vi) capture in structural materials, coolant, etc.

vii) escape from the reactor core.

In order to calculate the probabilities of any of these events occurring, it is necessary to consider the nuclear crosssections for the various processes. The important cross-sections for ${ }^{235} \mathrm{U}$ and

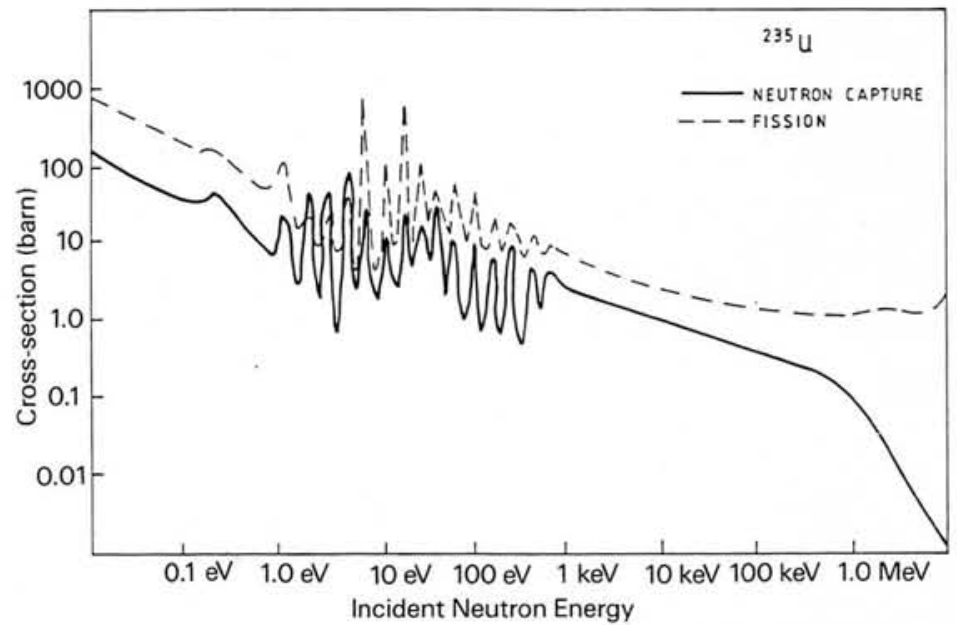

Fig. 1 - Neutron fission and capture cross-sections for ${ }^{235} \mathrm{U}$.

${ }^{238} \mathrm{U}$ are shown in Figs. 1 and 2 respectively. It can be seen that ${ }^{235} U$ has a significant fission cross-section at all neutron energies and particularly at low energies, whereas ${ }^{238} \mathrm{U}$ will only undergo fission when struck by a neutron with an energy greater than about $1 \mathrm{MeV}$. For this reason ${ }^{235} \mathrm{U}$ is referred to a fissile isotope and ${ }^{238} \mathrm{U}$ is referred to a fissionable isotope.

The cross-sections for neutron capture exhibit rapid changes with energy in the $10-1000 \mathrm{eV}$ region, particularly so for ${ }^{238} \mathrm{U}$. These changes are due to resonance effects and represent the very high neutron absorption probability when the energy of the incident neutron, together with its binding energy in the compound nucleus, coincide with an energy level of the compound nucleus.

Natural uranium contains $99.3 \%{ }^{238} \mathrm{U}$ and only $0.7 \%$ of the fissile isotope ${ }^{235} \mathrm{U}$. The high level of resonance capture in ${ }^{238} \mathrm{U}$ means that it is impossible to sustain a chain reaction in natural uranium alone. If, however, some means is found of slowing down the fast neutrons, while keeping them out of contact with uranium nuclei and hence avoiding a large amount of resonance capture, it is possible to sustain a chain reaction in natural uranium.

This can be achieved by lumping the fuel together in the form of fuel rods and surrounding them by a moderating material. The role of the moderator is simply to slow the neutrons down by a series of elastic collisions until the neutron energy is in equilibrium with that of atoms at ordinary temperatures. The neutrons are then described as thermal and have energies in the range of 0.01 to $0.1 \mathrm{eV}$. Reactors operating on this principle are called thermal reactors. Good moderating materials have a small mass, to maximise the neutron energy loss per collision, a high elastic scattering crosssection and a low capture cross-section.
Typical moderating materials used in reactors are water (both $\mathrm{H}_{2} \mathrm{O}$ and $\mathrm{D}_{2} \mathrm{O}$ ) and carbon, in the form of graphite.

Increasing the relative amount of fissile isotopes in the reactor fuel is another means of achieving a chain reaction and this process is referred to as enrichment of the fuel. If there is a suitably high fraction of fissile isotopes it is possible to sustain a chain reaction without the need for a moderating material. Reactors operating on this principle are referred to as fast reactors, as the bulk of fissions in them take place with fast neutrons, that is neutrons with energies higher than the resonance region.

\section{Factors Affecting Reactivity}

The proper and detailed analysis of the possible changes in reactivity, during normal operations and under all foreseeable accident conditions, is a vital part of the overall safety assessment carried out for every nuclear reactor. Nowadays these calculations are accomplished with the aid of large computer models of the reactor system, which are able to deal with the complex inter-relationships between the large number of factors that can affect the reactivity. Such models are essential in analysing the detailed spatial effects which are particularly important in some reactor designs. Nevertheless, some general understanding of the behaviour of a particular reactor can be obtained by considering in isolation some of the individual factors affecting reactivity.

\section{Scattering}

Elastic scattering causes neutrons to migrate in a random zig-zag manner, effectively increasing the distance that neutrons can travel within the reactor before escaping and being captured outside. Scattering, therefore, reduces the fraction of neutrons that leak from the reactor. Since only neutrons absorbed 


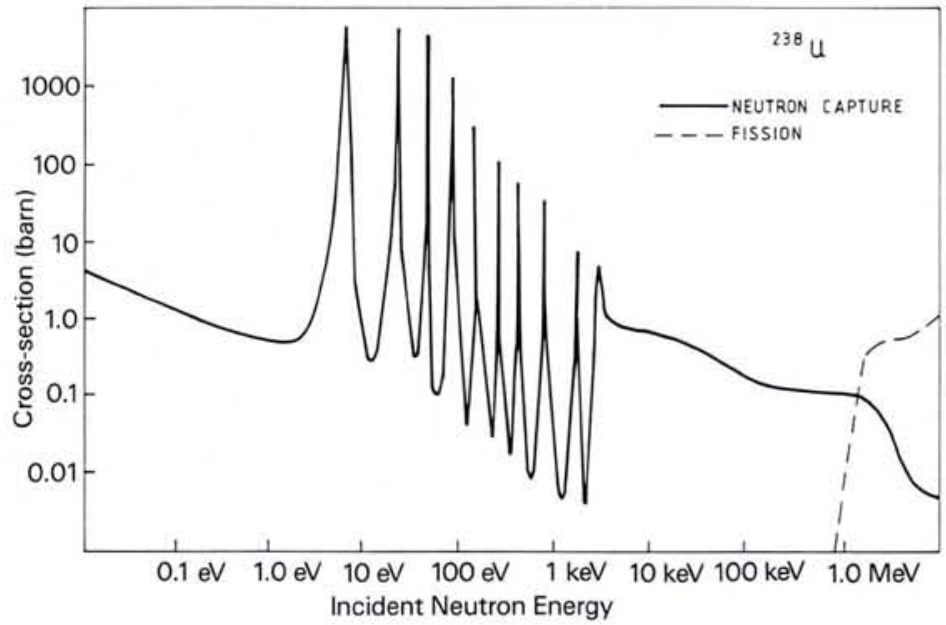

Fig. 2 - Neutron fission and capture cross-sections for ${ }^{238} \mathrm{U}$.

within the reactor's core have a chance of causing fission, the loss of a scattering material will, all other things being equal, tend to reduce reactivity.

\section{Moderation}

In general, an increase in the amount of moderation will lead to an increased proportion of thermal neutrons and this will in turn lead to an increase in reactivity. However, all practical moderators are also, to a greater or lesser extent, neutron absorbers and for these materials there is an optimum degree of moderation which gives the maximum reactivity. Beyond this level, an increase in moderator will produce a decrease in reactivity.

\section{Capture}

The loss of a pure capturing or absorbing material will tend to increase reactivity. Neutrons are either captured or cause fission and if the number captured is reduced the fraction causing fission will increase, with a corresponding increase in reactivity.

\section{Fission Products}

Fission products capture neutrons and as they build up during the operation of a reactor, the rate of neutron capture increases. To overcome this effect, additional fissile material is required. In terms of reactivity control, the most important fission product is ${ }^{135} \mathrm{Xe}$, which has a very high cross-section for the capture of neutrons and is referred to as a neutron poison. In a typical thermal reactor, about $2 \%$ of all neutrons are captured in ${ }^{135} \mathrm{Xe}$. Most of the ${ }^{135} \mathrm{Xe}$ arises from the radioactive decay of ${ }^{135} \mathrm{I}$, a common fission product with a half life of 6.7 hours, and ${ }^{135} \mathrm{Xe}$ itself decays with a half life of 9.2 hours. In steady operation the production and destruction of ${ }^{135} \mathrm{Xe}$ are in equilibrium and its population stabilises at a constant level dependent on the power. However, when reactor power is effect is fast acting and it provides a most important contribution to the inherent stability of reactors.

\section{Moderator Temperature}

An increase in moderator temperature causes the thermal neutrons to be shifted to slightly higher energies. If the only fissile material is ${ }^{235} \mathrm{U}$, higher energy thermal neutrons will have reduced fission cross-sections and the moderator temperature coefficient will thus be negative. In the case of ${ }^{239} \mathrm{Pu}$, which is a fissile isotope produced by neutron cap ture in ${ }^{238} \mathrm{U}$, there is a broad resonance in the fission cross-section at an energy of $0.3 \mathrm{eV}$. Any increase in moderator temperature causes more neutrons to be absorbed in this resonance with a consequent increase in reactivity. In some designs of thermal reactor, the ${ }^{239} \mathrm{Pu}$ effect can dominate and the overall moderator coefficient can be positive. However, there is a significant delay between changes in reactor power and changes in moderator temperature, particularly in large graphite moderated reactors.

\section{Fuel Temperature}

An increase in fuel temperature increases the relative thermal motion of the atoms in the fuel and the neutrons. Most reactor fuel contains significant amounts of ${ }^{238} \mathrm{U}$, and in this case the increased motion causes an effective broadening of the resonances in the ${ }^{238} \mathrm{U}$ capture cross-section. This effect is known as Doppler broadening and it results in more neutrons being captured in the resonances, with a corresponding decrease in reactivity. Increased fuel temperature also leads to a reduction in fuel density and this tends to reduce reactivity, although the effect is generally less important than that of Doppler broadening. Overall there is a strong negative fuel temperature coefficient and, furthermore, since fuel temperature and reactor power rise and fall together, with little or no time lag between them, the

\section{Coolant Voidage}

As the temperature of a reactor coolant is increased, its density will fall. In the case of a water cooled reactor, boiling will eventually take place. As the density of steam is much less than that of water, the production of steam can be considered as equivalent to producing tion of voids necessarily results in the expulsion of coolant material from the core and this will have an effect on the reactivity. In order to determine the socalled void coefficient it is necessary to consider the effect that a reduction in the amount of coolant will have in terms of scattering, moderation and capture. The relative importance of these three effects varies not only with reactor design but also with operating history. voids in the cooling water. The produc-

\section{Nantes}

\section{Visitor Positions - Nuclear Physics -}

Applications are being accepted for two visitor positions at Laboratoire de Spectroscopie Nucléaire - Université of NANTES (FRANCE). One opening is a research associate to work on Heavy-lon experimental or theoretical Nuclear-Physics. Our group is currently engaged in collaborations with the near GANIL National Facility. Full appointment will be for eight months or can be combined with original partial salary for longer period.

The second position will include, in addition, physics teaching responsibilities at the undergraduate level. Basic knowledge of the french language is required. Full appointment will be for one year with immediate opening and possible extension.

Send resumes and references promptly to

D. ARDOUIN - Faculté des Sciences - Université de NANTES

F - 44072 NANTES Cédex 03 - FRANCE

or call (33) 40373066 for further information. 


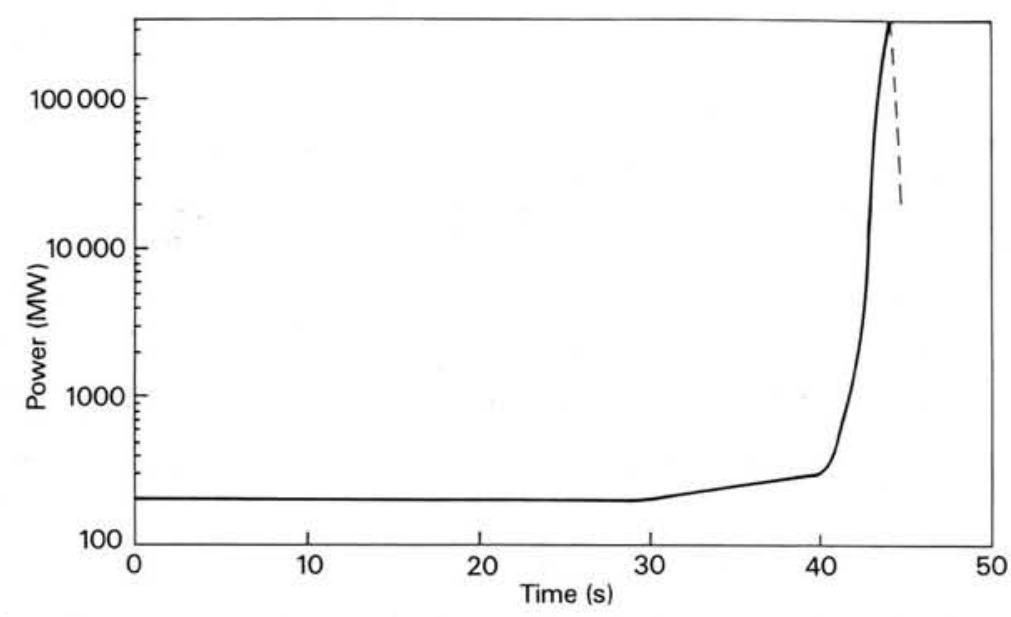

Fig. 3 - The power generated by the Chernobyl reactor in the period following the run down of the main coolant pumps. Initially $(0-30$ s) the reactivity increase caused by the positive void coefficient was counteracted by the automatic control rods but when they ceased to be effective, the power began to rise $(30-40 \mathrm{~s})$ following which, the reactor core became prompt critical and the power then rose to over 100 times its normal operating level in only a few seconds. At this point the fuel disintegrated.

\section{Power}

An increase in reactor power will, in the absence of any operational or corrective measures, cause temperatures to rise and densities to be reduced. The power coefficient, which relates changes in power to changes in reactivity, is therefore a combination of the temperature and density coefficients for the reactor fuel, coolant and moderator. A reactor with a negative power coefficient will have inherent stability and will automatically maintain constant power. On the other hand, a reactor that has a positive power coefficient would require continual adjustment in order to maintain the power constant.

\section{REACTOR DESIGNS}

In terms of overall reactor stability the power coefficient is the most important of the various reactivity coefficients and this is now discussed for a number of reactor designs, including the RBMK which was the type operated at Chernobyl.

\section{The PWR}

In the Pressurised Water Reactor ordinary ('light') water serves as both a reactor coolant and a moderator. The reactor fuel consists of uranium oxide enriched in ${ }^{235} U$ to about $3 \%$. Long term reactivity control is achieved by the use of a soluble neutron absorber (boric acid) in the coolant water and solid control rods are used for startup and shutdown as well as for short term reactivity control. In normal operation the high pressure maintained within the reactor ensures that boiling does not take place in the core of a PWR.

An increase in power and hence temperature of a PWR would cause the coo- lant water to expand and there would be a net loss of water from the reactor core, with three main effects:

i) a loss of moderator, tending to reduce reactivity;

ii) a loss of scattering, tending to reduce reactivity;

iii) a loss of capture, tending to increase reactivity.

In a typical large PWR, the leakage of neutrons from the core is small so the effect of (ii) is small. The PWR is designed so that the amount of moderation is less than the optimum value and any loss of moderator will lead to a reduction in reactivity. In other words, the loss of moderation by water outweighs the effects of a loss of capture in water. The PWR therefore has a negative void coefficient and together with a negative fuel temperature coefficient this ensures that the overall power coefficient is negative and the reactor core is inherently stable.

\section{The BWR}

The Boiling Water Reactor is, in terms of reactivity effects, very similar to the PWR, the difference being that the coolant water is allowed to boil within the reactor core, so that during normal operation there are voids in the coolant. The BWR has a negative void coefficient and this property is exploited in the system used for changing the power level.

\section{The AGR}

The Advanced Gas-cooled Reactor, built in the United Kingdom, uses $\mathrm{CO}_{2}$ gas as a coolant and graphite as moderator. The fuel consists of uranium oxide enriched in ${ }^{235} \mathrm{U}$ to about $2 \frac{1}{2} \%$.

An increase in the power of an AGR would rapidly increase the temperature of the fuel and the $\mathrm{CO}_{2}$ but would increase the graphite temperature very slowly. The $\mathrm{CO}_{2}$ has only a negligible effect on reactivity. The moderator temperature coefficient in an AGR is positive (except at the start of life when no ${ }^{239} \mathrm{Pu}$ is present). However, the moderator time constant is very long (of the order of 15 minutes) and this positive coefficient plays no part in determining the short term stability of the reactor. The negative fuel temperature coefficient ensures that over short periods of time the power coefficient is negative and the reactor is stable.

\section{The RBMK}

The Large Capacity Channel Reactor (RBMK in Russian) is cooled by boiling light water and moderated by graphite. The reactor fuel consists of uranium oxide enriched in ${ }^{235} U$ to $2 \%$.

In order to minimise the level of ${ }^{235} \mathrm{U}$ enrichment required, the RBMK designers chose an amount of graphite very close to the optimum value. A consequence of this is that, unlike the 'undermoderated' PWR and BWR designs, a change in the amount of coolant water in the core has relatively little effect on the level of moderation. In the event of water being lost from the core, the reduction in the amount of capture is the dominant effect and, therefore, the void coefficient is positive. Under normal operating conditions the RBMK's power coefficient is slightly negative, because the negative fuel temperature coefficient is just sufficient to outweigh the positive void coefficient. However, as the power level is reduced both the void and fuel temperature coefficients increase and, at power below $20 \%$ of full power, the power coefficient becomes positive. For this reason, the operating rules for the RBMK forbade sustained operation below $20 \%$ of full power.

As mentioned earlier, the effect of a reduction in the scattering provided by the cooling water is to increase the numbers of neutrons leaking from the core and hence to reduce reactivity. The presence of control absorber rods within the reactor core can be thought of as providing additional leakage boundaries, thereby enhancing this effect. Therefore, the more absorber rods there are in the core the more negative is the void coefficient. At Chernobyl the reactor was completing a period of full power operation and had been reduced to a level of about $7 \%$ of full power just prior to the accident. The level of ${ }^{135} \mathrm{Xe}$ in the core had built up and this, as well as a number of other factors, had led to the withdrawal of a large number of control rods from the core, in order to maintain 
criticality. This meant that the void coefficient was even more strongly positive and, as the power was well below the $20 \%$ level, so was the power coefficient.

In addition to the positive void and power coefficients, many of the safeguard systems had been deliberately disabled on the Chernobyl reactor just prior to the accident. When the turbogenerator experiment began, the main coolant pumps started to run down and the water in the core started to boil rapidly, increasing reactivity because of the positive void coefficient. Initially the automatic control rods were able to counteract this effect but soon they ceased to be effective and the reactivity and power began to increase. The positive power coefficient then caused the reactivity to rise until the reactor became prompt critical and the power increased at an extremely rapid rate, rising to about 100 times normal full power in only a few seconds (see Fig. 3).

Analyses carried out since the accident have indicated two factors which may have added to the severity of the power rise.

i) The RBMK absorber rods had graphite 'follower' rods suspended beneath them to increase their effectiveness. In normal operation, as the absorber rods are inserted into the core they replace the graphite rod. Without the graphite rod, the absorber rod would be replacing water (which is itself a good absorber) and it would not be so effective. At Chernobyl, the neutron flux was peaked at the top and the bottom of the core, due partly to the ${ }^{135} \mathrm{Xe}$ build up in the core centre. When the Chernobyl operators saw the initial power rise they pushed the manual reactor trip button, causing the absorbers to be inserted into the core. It has been suggested that as the graphite followers displaced water in the lower portion of the core where the neutron flux was high, they produced a local increase in reactivity.

ii) It has alternatively been suggested that a positive reactivity insertion in the lower part of the core could have been caused by cavitation in the main coolant pumps which resulted in the injection of vapour bubbles into the core.

\section{Summary}

The accident at Chernobyl has highlighted many issues concerned with the safety of nuclear reactors including that of reactivity coefficients. The above summarizes the reasons for their importance and indicates why the inherent stability of a power reactor depends on its specific design.

\section{Astronomers in Europe}

\section{Dear Sir,}

The front-page Editorial in the April 1987 issue of Europhys. News (which I read only now owing to a two-year absence in the USA) contains a rather categorical statement $(p .46)$ to the effect that a broad consensus exists among European astronomers in favour of forming a European Astronomical Society (EAS), and that the only reason preventing this desirable development is that they (we) are too miserly to pay for it.

In fact, most of us are members of the International Astronomical Union, which has a regular series of European Regional Meetings. Since 1969, we do have a common European journal, Astronomy and Astrophysics. Those of us, including yours truly, who like to feel some kinship with the (other) physicists in our departments, can adhere to EPS and its Astronomy and Astrophysics Division at minimum rates if we are members of our national physical society or one of the

\section{The Chairman of the A \& A Division Replies}

This letter is a welcome reaction to the plans for a European Astronomical Society. The creation of such a society has been on the agenda of the Astronomy and Astrophysics Board of the EPS for some time - and it has proved to be a rather complex issue.

Dr. Andersen thinks that the financial aspects of such an endeavour have been stressed too much in the report on the Council meeting and complains that astronomers are unfairly depicted as having - to say the least - a very economical attitude in personal matters. This may be so.

The primary reason, why astronomers have not joined EPS in great numbers is probably rather that they do not quite identify with physicists. Most of them belong to national astronomical societies and some also belong to the American Astronomical Society. More established astronomers can seek approval of their National Academies to become members of the International Astronomical Union (IAU).

Given the current state and outlook of astronomy in Europe and given the traditional links between European astronomers - in the East and the West - the Astronomy and Astrophysics Board of the EPS has recommended that an independent, representative organisation for European astronomers (with individual members) be founded. The Board re- astronomical societies that is a Collaborating Society. We are kept very well informed, also through this bulletin, of an already overwhelming multitude of interesting meetings. But, perhaps because the community is so much smaller, many subjects could simply not be adequately discussed in a purely European audience.

I suggest that, rather than labelling astronomers wholesale as being even more avaricious, narrow-minded, and disloyal than their "real" physicist brethren who are dealt with in no uncertain terms in the following paragraphs of the Editorial, the EPS should explain to us why forming an EAS would be worth our time and, yes, money. That approach might not be less successful.

\section{Sincerely yours,}

\section{Johannes Andersen}

Copenhagen University Observatory, Denmark

gards this as a cultural need. Such a society can provide many services, as, for example organise discussion meetings and workshops with a substantial attendance of young astronomers. Its annual meetings could probably be held within the current framework of the European Regional Astronomy Meetings of the IAU (which are co-sponsored by the EPS through its A \& A Division), since one should try not to create any more large general conferences. The continuity provided by a dedicated European Astronomical Society will in turn assure a more uniform, high standard for the Regional Astronomy Meetings.

If such a society is run on an honorary basis exclusively, the membership fee can be kept very low. Collaboration can be sought with those national astronomical societies that deem this desirable as well as with the EPS. Astronomers could then also become Individual Ordinary Members of the EPS at minimum rates. Given this scenario, one might wonder why a European Astronomical Society is not yet in existence.

Martin C.E. Huber (Chairman, EPS Astronomy \& Astrophysics Division)

The strictures on parsimony and parasiting were not aimed specifically, or even mainly, at the astrophysicists. The juxtaposition of the two paragraphs was unfortunate and for this we apologise. Ed. 